\title{
Emergence of diffusion in finite quantum systems
}

\author{
M. Esposito and P. Gaspard \\ Center for Nonlinear Phenomena and Complex Systems, Université Libre de Bruxelles, Code Postal 231, Campus Plaine, \\ B-1050 Brussels, Belgium
}

(Received 31 August 2004; revised manuscript received 27 December 2004; published 14 June 2005)

\begin{abstract}
We study the emergence of diffusion for a quantum particle moving in a finite and translationally invariant one-dimensional subsystem described by a tight-binding Hamiltonian with a single energy band and interacting with its environment by an interaction energy proportional to some coupling parameter. We show that there exists a crossover between a nondiffusive relaxation regime for small sizes or low values of the coupling parameter and a diffusive regime above a critical size or for higher values of the coupling parameter. In the nondiffusive regime, the relaxation is characterized by oscillations decaying at rates independent of the size and proportional to the square of the coupling parameter and the temperature of the environment. In the diffusive regime, the damped oscillations have disappeared and the relaxation rate is inversely proportional to the square of the size. The diffusion coefficient is proportional to the square of the energy bandwidth of the subsystem and inversely proportional to the temperature of the environment and the square of the coupling parameter. The critical size where the crossover happens is obtained analytically.

DOI: 10.1103/PhysRevB.71.214302 PACS number(s): 66.30. $-\mathrm{h}, 05.60 .-\mathrm{k}, 72.10 .-\mathrm{d}, 73.23 .-\mathrm{b}$
\end{abstract}

\section{INTRODUCTION}

Atoms, molecules, or spins coupled to their environment undergo decay processes in the populations and coherences of their quantum states. ${ }^{1-6}$ In the weak-coupling limit, the rates of decay are given by Fermi's golden rule and vanish as the square of the coupling parameter. On the other hand, there exist other irreversible processes such as the diffusion of particles. In this case, their random walk results from the perturbations of the environment hindering the ballistic motion of the particles. The pioneering work by Einstein in 1905 has shown that diffusion is intimately related to conduction or mobility. ${ }^{7}$ Since then, diffusion has been studied in classical ${ }^{8-11}$ as well as quantum systems. ${ }^{12-27}$ Diffusion is sustained in large spatially extended systems but is absent in such small systems as atoms where simple decay processes prevail. We may thus wonder how the transport property of diffusion can emerge as the size of the system increases. This question is of special importance in nanoscience which provides us with systems of intermediate sizes between the atoms and macroscopic systems. Examples of nanosystems sustaining transport of electric charges are the onedimensional molecular chains such as conducting polymers ${ }^{28}$ and carbon nanotubes, ${ }^{29}$ which may form closed loops of different sizes. This raises the question of the minimum size above which diffusion can appear in such nanosystems.

The purpose of the present paper is to understand how diffusion can emerge in closed-loop molecular chains as their size increases. The vehicle of our study is a model in which a quantum particle moves along a one-dimensional chain forming a closed loop. This motion is described by a tightbinding Hamiltonian with one energy band and which is coupled to a fast thermal environment in the subsystem-plusreservoir approach. This model is invariant under spatial translations along the one-dimensional chain, which allows us to recover the transport property of diffusion in the large- system limit. In the weak-coupling limit, this model has the advantage of being described by a quantum master equation which is exactly solvable.

The plan of the paper is the following. Our translationally invariant model is defined in Sec. II. The dynamics of this system is ruled by a Redfield quantum master equation. ${ }^{2-6} \mathrm{As}$ explained in Sec. III, the long-time evolution can be studied in terms of the eigenvalues and the associated eigenstates of the Redfield superoperator. This provides us with a criterion for the emergence of diffusion in the system and allows us to determine the size where diffusion appears. We show in Sec. IV that diffusion dominates the long-time dynamics for sizes larger than a minimum one or, equivalently, if the coupling parameter is large enough. Conclusions are drawn in Sec. V.

\section{DEFINING THE SYSTEM}

\section{A. Subsystem}

Let us consider a quantum particle moving in a onedimensional periodic potential. Such translationally invariant subsystems are known to present an energy spectrum with a band structure. We suppose that the lower-energy band does not overlap with the higher-energy bands. We denote $\Delta E_{\text {band }}$ the energy spacing between the lower band in the spectrum and the next one at higher energies. We assume that the thermal fluctuations $k_{\mathrm{B}} T$, due to the coupling to the environment, are small compared to $\Delta E_{\text {band }}$. The subsystem dynamics is therefore restricted to the lower-energy band. In this case, the motion of the quantum particle only occurs by the tunneling of the particle through the potential barriers separating the lattice sites. This subsystem can be described by the Hamiltonian 


$$
\hat{H}_{\mathrm{s}}=\left(\begin{array}{ccccccc}
E_{0} & -A & 0 & 0 & \ldots & 0 & -A \\
-A & E_{0} & -A & 0 & \ldots & 0 & 0 \\
0 & -A & E_{0} & -A & & 0 & 0 \\
\vdots & & \ddots & \ddots & \ddots & & \vdots \\
0 & 0 & & -A & E_{0} & -A & 0 \\
0 & 0 & \ldots & 0 & -A & E_{0} & -A \\
-A & 0 & \ldots & 0 & 0 & -A & E_{0}
\end{array}\right)_{N \times N}
$$

represented in the site basis $|l\rangle$, where $l$ takes the values $l$ $=0,1, \ldots, N-1 . N$ is the length of the chain. We have here chosen periodic (Born-van Karman) boundary conditions because the chain is supposed to form a closed loop.

Such a Hamiltonian, often called tight-binding or Hückel Hamiltonian, is a simple model of a spatially periodic subsystem and has therefore a wide range of applications. The tight-binding Hamiltonian is for instance commonly used in solid-state physics to describe the electronic band structure of weakly interacting solids ${ }^{7}$ and in polymer physics to describe electronic conduction along the polymer backbone or polymer optical properties, ${ }^{28}$ as well as to understanding the conduction properties of carbon nanotubes. ${ }^{29}$ This Hamiltonian describes a process of quantum tunneling from site to site. The parameter $A$ is given in terms of the overlap between the wave functions localized at the sites and is proportional to the quantum amplitude of tunneling.

The stationary Schrödinger equation of the tight-binding Hamiltonian is given by

$$
\hat{H}_{\mathrm{s}}|k\rangle=\epsilon_{k}|k\rangle,
$$

where the eigenvalues are

$$
\epsilon_{k}=E_{0}-2 A \cos k \frac{2 \pi}{N}
$$

and the eigenvectors

$$
\langle l \mid k\rangle=\frac{1}{\sqrt{N}} \mathrm{e}^{i l k 2 \pi / N},
$$

with $k=0,1, \ldots, N-1$. The closure relation is given by

$$
\frac{1}{N} \sum_{l=0}^{N-1} \mathrm{e}^{i l\left(k-k^{\prime}\right) 2 \pi / N}=\delta_{k k^{\prime}}
$$

Accordingly, the energy spectrum of the Hamiltonian (1) contains a single energy band of width $4 A$ and the motion of the particle would be purely ballistic without coupling to a fluctuating environment.

\section{B. Coupling to the environment}

We now suppose that the subsystem is embedded in a large environment.

The Hamiltonian of the total system composed of the onedimensional chain and its environment (or heat bath) is given by

$$
\hat{H}_{\mathrm{tot}}=\hat{H}_{\mathrm{s}}+\hat{H}_{\mathrm{b}}+\lambda \sum_{l} \hat{S}_{l} \hat{B}_{l}
$$

where $\hat{H}_{\mathrm{b}}$ is the environment Hamiltonian, $\hat{S}_{l}$ the subsystem coupling operators, $\hat{B}_{l}$ the environment coupling operators, and $\lambda$ the coupling parameter which measures the intensity of the interaction between the subsystem and its environment. We adopt the convention that $\hbar=1$.

The reduced dynamics for the density matrix $\hat{\rho}(t)$ $=\operatorname{Tr}_{\mathrm{b}} \hat{\rho}_{\text {tot }}(t)$ of the subsystem is known to obey a Redfield quantum master equation for weak coupling to the environment. $^{2-6}$ This equation can be systematically derived from the complete von Neumann equation for the total system by second-order perturbation theory and its validity has been abundantly tested in many different contexts. ${ }^{30-32}$ On time scales longer than the correlation time of the environment, the Redfield quantum master equation is Markovian and reads

$$
\begin{aligned}
\frac{d \hat{\rho}}{d t}= & \hat{\hat{\mathcal{L}}}_{\mathrm{Red}} \hat{\rho}=-i\left[\hat{H}_{\mathrm{s}}, \hat{\rho}\right] \\
& +\lambda^{2} \sum_{l}\left(\hat{T}_{l} \hat{\rho} \hat{S}_{l}+\hat{S}_{l}^{\dagger} \hat{\rho} \hat{T}_{l}^{\dagger}-\hat{S}_{l} \hat{T}_{l} \hat{\rho}-\hat{\rho} \hat{T}_{l}^{\dagger} \hat{S}_{l}^{\dagger}\right)+O\left(\lambda^{3}\right),
\end{aligned}
$$

where $\hat{\hat{\mathcal{L}}}_{\text {Red }}$ is called the Redfield superoperator and

$$
\hat{T}_{l} \equiv \sum_{l^{\prime}} \int_{0}^{\infty} d \tau \alpha_{l l^{\prime}}(\tau) \mathrm{e}^{-i \hat{H}_{\mathrm{s}} \tau} \hat{S}_{l^{\prime}} \mathrm{e}^{i \hat{H}_{\mathrm{s}} \tau}
$$

The correlation function of the environment which contains all the necessary information to describe the coupling of the subsystem to its environment is given by

$$
\alpha_{l l^{\prime}}(\tau)=\operatorname{Tr}_{\mathrm{b}} \hat{\rho}_{\mathrm{b}}^{\mathrm{eq}} \mathrm{e}^{i \hat{H}_{\mathrm{b}} \tau} \hat{B}_{l} \mathrm{e}^{-i \hat{H}_{\mathrm{b}} \tau} \hat{B}_{l^{\prime}}
$$

where $\hat{\rho}_{\mathrm{b}}^{\text {eq }}$ is the canonical equilibrium state of the environment.

Let us now specify the interaction of the subsystem with its environment. We define the subsystem coupling operators as

$$
\left\langle l^{\prime}\left|\hat{S}_{l}\right| l^{\prime \prime}\right\rangle=\delta_{l l^{\prime}} \delta_{l l^{\prime \prime}}
$$

These operators are diagonal in the site basis of the subsystem taking the unit value if the particle is located on the site $l$ and zero otherwise.

Now, we need to specify the correlation functions. We assume in our model that the correlation time of the environment $\left(\tau_{\mathrm{b}}=1 / \omega_{\mathrm{b}}\right)$ is short compared to the shortest time scale of the subsystem $\left(\tau_{\mathrm{s}}=\pi / 2 A\right)$. Furthermore, we suppose that the range of the spatial correlations of the environment fluctuations is shorter than the distance between two neighboring lattice sites of the subsystem: $\alpha_{l l^{\prime}}(\tau) \simeq \alpha(\tau) \delta_{l l^{\prime}}$. We can therefore rewrite Eq. (8) as 


$$
\hat{T}_{l} \simeq \hat{S}_{l} \int_{0}^{\infty} d \tau \alpha(\tau) \equiv Q \hat{S}_{l} .
$$

If we also assume that the environment is Ohmic and that its temperature is large enough so that the thermal time $t_{\text {th }}$ $=1 / k_{\mathrm{B}} T$ is shorter than its correlation time $\tau_{\mathrm{b}}$, we find that the constant $Q$ characterizing the interaction of the subsystem with the environment is proportional to the temperature:

$$
Q=\pi K k_{\mathrm{B}} T,
$$

(see Appendix A).

Accordingly, under the assumptions

$$
t_{\text {th }} \ll \tau_{\mathrm{b}} \ll \tau_{\mathrm{s}},
$$

the Redfield equation of our model takes the final form

$$
\begin{aligned}
\frac{d \hat{\rho}}{d t}= & \hat{\hat{\mathcal{L}}}_{\mathrm{Red}} \hat{\rho}=-i\left[\hat{H}_{\mathrm{s}}, \hat{\rho}\right] \\
& +\lambda^{2} Q \sum_{l}\left(2 \hat{S}_{l} \hat{\rho} \hat{S}_{l}-\hat{S}_{l}^{2} \hat{\rho}-\hat{\rho} \hat{S}_{l}^{2}\right)+O\left(\lambda^{3}\right) .
\end{aligned}
$$

It can easily be verified by projecting this equation onto the site basis that it is translationally invariant (shifting all the site indices appearing in the projected equation by a constant does not modify the equation). Furthermore, this equation preserves the complete positivity of the density matrix because it has the Lindblad form ${ }^{33}$ which is the result of a coupling with delta correlation functions. ${ }^{6}$

\section{DIAGONALIZING THE REDFIELD SUPEROPERATOR}

The eigenvalues $s_{\nu}$ and associated eigenstates $\hat{\rho}^{\nu}$ of the Redfield superoperator are defined by

$$
\hat{\hat{\mathcal{L}}}_{\text {Red }} \hat{\rho}^{\nu}=s_{\nu} \hat{\rho}^{\nu},
$$

where $\nu$ is a set of parameters labeling the eigenstates. Because the Redfield superoperator is not anti-Hermitian, its eigenvalues can be complex numbers with a nonzero real part. The eigenvalue problem of the Redfield superoperator is important because the time evolution of the quantum master equation can then be decomposed onto the basis of the eigenstates as

$$
\hat{\rho}(t)=\mathrm{e}^{\hat{\hat{\mathcal{R}}}_{\mathrm{Red}} t} \hat{\rho}(0)=\sum_{\nu=1}^{N^{2}} c_{\nu}(0) \mathrm{e}^{S_{\nu} t} \hat{\rho}^{\nu}
$$

The dynamics is therefore given by a linear superposition of exponential or oscillatory exponential functions of time, which describes the relaxation toward the stationary equilibrium state. Since the reduced density matrix of the subsystem has $N^{2}$ elements, there is a total of $N^{2}$ eigenvalues and associated eigenstates.

\section{A. Bloch theorem for the density matrix}

Since the system is invariant under spatial translations, we can apply the Bloch theorem to the eigenstates of the Red- field superoperator. Thanks to this theorem, the state space of the superoperator can be decomposed into independent superoperators acting onto decoupled sectors associated with a Bloch number, also called the wave number. ${ }^{7}$ We emphasize that the Bloch theorem is here applied to density matrices instead of wave functions.

Let us define the superoperator $\hat{\hat{\mathcal{T}}}_{a}$ of the spatial translation by $a$ sites along the system ( $a$ is an integer) as

$$
\sum_{m, m^{\prime}}\left(\hat{\hat{\mathcal{T}}}_{a}\right)_{l l^{\prime}, m m^{\prime}} \rho_{m m^{\prime}}^{\nu}=\rho_{l+a, l^{\prime}+a}^{\nu}
$$

where we use the notation

$$
\left\langle l|\hat{\rho}| l^{\prime}\right\rangle=\rho_{l l^{\prime}} .
$$

This superoperator must have the group property

$$
\hat{\hat{\mathcal{T}}}_{a} \hat{\hat{\mathcal{T}}}_{a^{\prime}}=\hat{\hat{\mathcal{T}}}_{a^{\prime}} \hat{\hat{\mathcal{T}}}_{a}=\hat{\hat{\mathcal{T}}}_{a+a^{\prime}} .
$$

Because of the translational symmetry of the system, the translation superoperators commute with the Redfield superoperator:

$$
\hat{\hat{\mathcal{T}}}_{a} \hat{\hat{\mathcal{L}}}_{\mathrm{Red}} \hat{\rho}(t)=\hat{\hat{\mathcal{L}}}_{\text {Red }} \hat{\hat{\mathcal{T}}}_{a} \hat{\rho}(t) .
$$

Therefore, the Redfield superoperator and the translation superoperators have a basis of common eigenstates. If $\tau(a)$ denotes the eigenvalues of the translation superoperator, we have that

$$
\hat{\hat{\mathcal{T}}}_{a} \hat{\boldsymbol{\rho}}^{\nu}=\tau(a) \hat{\boldsymbol{\rho}}^{\nu}=\mathrm{e}^{i q a} \hat{\rho}^{\nu},
$$

where $q$ is the Bloch number, also called the wave number. In the site basis, Eq. (21) becomes

$$
\rho_{l+a, l^{\prime}+a}^{\nu}=\mathrm{e}^{i q a} \rho_{l l^{\prime}}^{\nu} .
$$

A useful consequence is that

$$
\rho_{l l^{\prime}}^{\nu}=\mathrm{e}^{i q l} \rho_{0, l^{\prime}-l^{\prime}}^{\nu} .
$$

In order to determine the allowed values of the Bloch number, we write by using Eq. (4) such that

$$
\rho_{l l^{\prime}}^{\nu}=\frac{1}{N} \sum_{k, k^{\prime}}\left\langle k\left|\hat{\rho}^{\nu}\right| k^{\prime}\right\rangle \mathrm{e}^{i\left(l k-l^{\prime} k^{\prime}\right) 2 \pi / N}
$$

and

$$
\rho_{l+1, l^{\prime}+1}^{\nu}=\frac{1}{N} \sum_{k, k^{\prime}}\left\langle k\left|\hat{\rho}^{\nu}\right| k^{\prime}\right\rangle \mathrm{e}^{i\left(l k-l^{\prime} k^{\prime}\right) 2 \pi / N} \mathrm{e}^{i\left(k-k^{\prime}\right) 2 \pi / N} .
$$

Due to Eq. (22), we also have

$$
\rho_{l+1, l^{\prime}+1}^{\nu}=\mathrm{e}^{i q} \rho_{l l^{\prime}}^{\nu} .
$$

Multiplying both sides of Eqs. (25) and (26) by $\left\langle l^{\prime} \mid k^{\prime \prime \prime}\right\rangle$ $\times\left\langle k^{\prime \prime} \mid l\right\rangle$, taking the sum $\Sigma_{l, l^{\prime}}$ of it, and identifying them, we get 


$$
\mathrm{e}^{i q}\left\langle k\left|\hat{\rho}^{\nu}\right| k^{\prime}\right\rangle=\mathrm{e}^{i\left(k-k^{\prime}\right) 2 \pi / N}\left\langle k\left|\hat{\rho}^{\nu}\right| k^{\prime}\right\rangle .
$$

Now, we notice that if $q \neq\left(k-k^{\prime}\right) 2 \pi / N$, then $\left\langle k\left|\hat{\rho}^{\nu}\right| k^{\prime}\right\rangle=0$. Using finally the periodicity

$$
\begin{aligned}
& \rho_{l+N, l^{\prime}}^{\nu}=\rho_{l l^{\prime}}^{\nu}, \\
& \rho_{l, l^{\prime}+N}^{\nu}=\rho_{l l^{\prime}}^{\nu},
\end{aligned}
$$

we find with Eq. (22) that the Bloch number takes the values

$$
q=j \frac{2 \pi}{N}, \quad \text { where } j=0,1, \ldots, N-1 .
$$

Accordingly, the Redfield superoperator can be blockdiagonalized into $N$ independent blocks, which each contains $N$ eigenvalues as we shall see in the following.

\section{B. Simplifying the problem}

We will now formulate the eigenvalue problem of the Redfield superoperator in each sector labeled by a given wave number $q$.

For this purpose, we project Eq. (15) onto the site basis using the explicit expression (14) of the Redfield superoperator. We get

$$
\begin{aligned}
s_{\nu} \rho_{l l^{\prime}}^{\nu}= & -i A\left(-\rho_{l-1, l^{\prime}}^{\nu}-\rho_{l+1, l^{\prime}}^{\nu}+\rho_{l, l^{\prime}-1}^{\nu}+\rho_{l, l^{\prime}+1}^{\nu}\right) \\
& +2 \lambda^{2} Q\left(\delta_{l l^{\prime}}-1\right) \rho_{l l^{\prime}}^{\nu} .
\end{aligned}
$$

Using Eq. (23) and replacing $l^{\prime}-l$ by $l$, we get

$$
\begin{aligned}
\left(s_{\nu}+2 Q \lambda^{2}\right) \rho_{0 l}^{\nu}= & 2 A\left(\sin \frac{q}{2}\right)\left(\mathrm{e}^{-i q / 2} \rho_{0, l+1}^{\nu}-\mathrm{e}^{i q / 2} \rho_{0, l-1}^{\nu}\right) \\
& +2 Q \lambda^{2} \rho_{00}^{\nu} \delta_{0 l} .
\end{aligned}
$$

Making the change of variable

$$
\rho_{0 l}^{\nu}=i^{-l} \mathrm{e}^{i(q / 2) l} f_{l},
$$

we obtain the simpler eigenvalue equation

$$
\mu_{l} f_{l}=\delta_{0 l} f_{l}-i \beta\left(f_{l-1}+f_{l+1}\right),
$$

where

$$
\mu_{\nu}=\frac{s_{\nu}}{2 Q \lambda^{2}}+1
$$

and

$$
\beta=\frac{A}{Q \lambda^{2}} \sin \frac{q}{2} \text {. }
$$

\section{Spectrum of Redfieldian eigenvalues}

We can write the expression (34) in matrix form (we no longer write the index $\nu$ to simplify the notation)

$$
\mu \vec{f}=\hat{W} \vec{f},
$$

where $\mu$ are the eigenvalues, $\vec{f}=\left(f_{0}, \ldots, f_{N-1}\right)$ the eigenvectors of size $N$, and $\hat{W}$ the $N \times N$ matrix

$$
\left(\begin{array}{ccccccc}
1 & -i \beta & & & & & -i \beta i^{-N} \mathrm{e}^{i N q / 2} \\
-i \beta & 0 & -i \beta & & & & \\
& -i \beta & 0 & -i \beta & & & \\
& & \ddots & \ddots & \ddots & & \\
& & & -i \beta & 0 & -i \beta & \\
-i \beta i^{N} \mathrm{e}^{-i N q / 2} & & & & & & \\
& & & & & -i \beta & 0
\end{array}\right)
$$

where the matrix elements in the off-diagonal corners guarantee the periodic boundary conditions (28) and (29). We have therefore that $f_{l}=f_{l+N}$.

The complete spectrum of the $N^{2}$ eigenvalues of the Redfield superoperator has been calculated by the numerical diagonalization of Eq. (38) and is depicted in Fig. 1 as a function of the wave number $q$.

In Fig. 1(a), we depict the spectrum in the case $A / Q \lambda^{2}$ $<\frac{1}{2}$. In this case, we observe two families of eigenvalues. A branch containing a single eigenvalue for each value of the wave number $q$ and connected to $\mu=1$ when the wave number vanishes. According to Eq. (35), the value $\mu=1$ corresponds to the eigenvalue $s=0$ of the stationary equilibrium state. Moreover, this branch is seen in Fig. 1(a) to approach $\mu=1$ with a quadratic dependence on the wave number $q$ as

$$
s^{(\mathrm{D})}=-D q^{2}+O\left(q^{4}\right) .
$$

As confirmed by the following analysis, this behavior shows that these eigenvalues are responsible for diffusion in the system and $D$ is the diffusion coefficient which can be calculated analytically as shown here below.

Beside the $N$ eigenvalues of the branch of diffusion, we also find many other eigenvalues, precisely $N^{2}-N$ of them, which are located slightly above the plane $\operatorname{Re} \mu=0$ with a nonvanishing imaginary part for most of them. Here, Eq. 

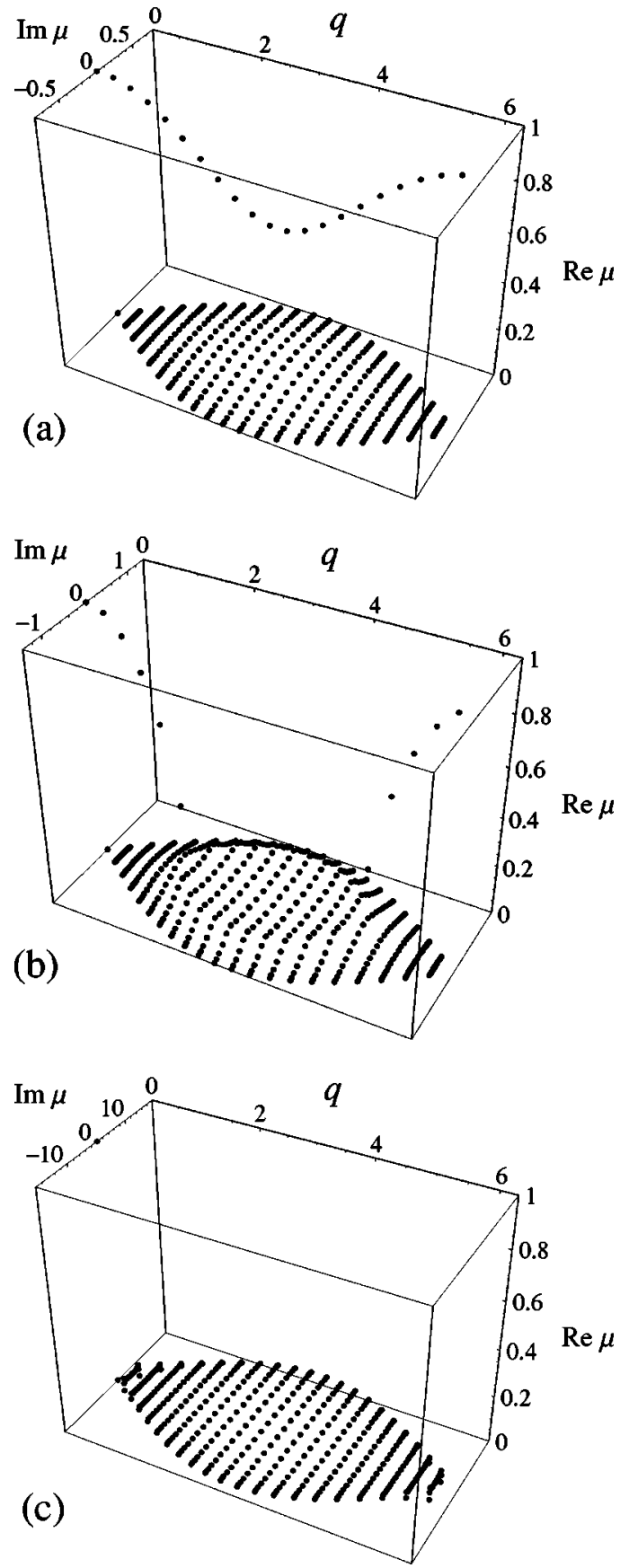

FIG. 1. Complete spectrum of the eigenvalues $\mu_{q \theta}$ related to the eigenvalues of the Redfield superoperator by Eq. (50). The system size is here $N=21$. In (a), $A / Q \lambda^{2}=0.4$. In (b), $A / Q \lambda^{2}=0.7$. In (c), $A / Q \lambda^{2}=10$.

(35) shows that the real part of these eigenvalues is given by

$$
\operatorname{Re} s^{(\mathrm{C})} \simeq-2 Q \lambda^{2} .
$$

Therefore, these eigenvalues describe decays at rates which are approximately equal to $2 Q \lambda^{2}$. Since they behave as $\lambda^{2}$, they can be explained by Fermi's golden rule. The eigenvalues with a nonvanishing imaginary part are associated with damped oscillations. The imaginary parts are essentially the Bohr frequencies of the Hamiltonian subsystem (1). We may thus interpret most of these eigenvalues as responsible for the process of decoherence of the quantum coherences. Since they have larger decay rates than the eigenvalues of the diffusive branch, they correspond to faster transients so that the long-time dynamics will be dominated in the case of Fig. 1(a) by diffusion, as shown in the following.

On the other hand, if $A / Q \lambda^{2}>\frac{1}{2}$, the diffusive eigenvalues disappear for some value of the wave number, as observed in Fig. 1(b). For very large values of $A / Q \lambda^{2}>\frac{1}{2}$, the diffusive branch of the spectrum is reduced to the sole eigenvalue at $q=0$, as seen in Fig. 1(c). In this case, diffusion has disappeared from the spectrum which only contains eigenvalues associated with damped oscillatory behavior (coherent eigenvalues). Hence diffusion can be supposed to have disappeared when its last nonzero eigenvalue has disappeared as in Fig. 1(c). This happens if the parameter $A / Q \lambda^{2}$ is large enough, as observed in Fig. 1. In order to obtain the precise value of this crossover, we need analytical expressions for the eigenvalues. This is the purpose of the next subsection.

\section{Analytical expressions for the eigenvalues}

The eigenvalues of the diffusive branch are obtained by going back to the eigenvalue equation (34) and assuming that the diffusive eigenvector satisfies $f_{l}=f_{-l}$.

If $l=0$ in Eq. (34) and using $f_{1}=f_{-1}$, we get

$$
\frac{1-\mu}{2}=i \beta \frac{f_{1}}{f_{0}}
$$

For $l \neq 0$, Eq. (34) gives

$$
\frac{f_{l}}{f_{l-1}}=\frac{-i \beta}{\mu+i \beta \frac{f_{l+1}}{f_{l}}} .
$$

Using Eqs. (41) and (42), we obtain by recurrence the continuous fraction

$$
\frac{1-\mu}{2}=i \beta \frac{-i \beta}{\mu+i \beta \frac{-i \beta}{\mu+i \beta \frac{-i \beta}{\mu+\cdots}}} .
$$

Defining $x \equiv(1-\mu) / 2$, Eq. (43) becomes

$$
x=i \beta \frac{-i \beta}{\mu+x} .
$$

By solving $x^{2}+\mu x-\beta^{2}=0$, we finally find that

$$
\mu=\mu^{(\mathrm{D})}=\sqrt{1-(2 \beta)^{2}},
$$

which is real if $\beta<1 / 2$. According to Eq. (35), the corresponding eigenvalue of the Redfield superoperator is given by

$$
s^{(\mathrm{D})}=-2 Q \lambda^{2}+2 Q \lambda^{2} \sqrt{1-(2 \beta)^{2}} .
$$

The dependence on the wave number is given by Eq. (36) as 


$$
s^{(\mathrm{D})}=-2 Q \lambda^{2}+2 Q \lambda^{2} \sqrt{1-\left(\frac{2 A}{Q \lambda^{2}} \sin \frac{q}{2}\right)^{2}},
$$

with the wave number $q$ taking the discrete values (30). We notice that possible finite-size corrections are exponentially small in the chain length $N$ and negligible. ${ }^{34}$ Expanding Eq. (47) in powers of the wave number, we find the dispersion relation (39) of diffusion with the diffusion coefficient

$$
D=\frac{A^{2}}{Q \lambda^{2}} .
$$

This result shows that the diffusion coefficient of the present model is proportional to the square of the width $4 A$ of the energy band and inversely proportional to the constant (12) characterizing the interaction of the subsystem with its environment and the square of the coupling parameter $\lambda$. The diffusion coefficient increases as the coupling to the environment vanishes. Indeed, in the absence of coupling, the quantum particle follows a ballistic motion on the chain and the diffusion coefficient is infinite in this limit. The environment hinders the ballistic motion by its thermal fluctuations. As shown in Appendix B, the diffusion coefficient is inversely proportional to the temperature and the conductivity of the model inversely proportional to the square of the temperature as expected.

Beside the diffusive eigenvalue, there exist the other family of eigenvalues responsible for simple decay processes. To derive the other family of eigenvalues, we assume instead that the eigenvectors of Eq. (34) takes the form $f_{l}=\mathrm{e}^{i \theta l}$ and that the diagonal element $l=l^{\prime}=0$ which is equal to unity is negligible. This assumption is confirmed numerically and has also been verified analytically. ${ }^{34}$ Accordingly, the corresponding eigenvalues are given by

$$
\mu^{(\mathrm{C})}=2 i \beta \cos \theta,
$$

where $\theta$ is a real number. Therefore, we now have that

$$
s^{(\mathrm{C})}=-2 Q \lambda^{2}+i 4 Q \lambda^{2} \beta \cos \theta
$$

or, equivalently,

$$
s^{(\mathrm{C})}=-2 Q \lambda^{2}+i 4 A \sin \frac{q}{2} \cos \theta,
$$

in terms of the wave number $q$ given by Eq. (30). Since this family contains $N^{2}-N$ eigenvalues, the angle $\theta$ also takes about $N$ discrete values. We see that these eigenvalues have a real part giving damping rates equal to $2 Q \lambda^{2}$ independently of the wave number $q$, as numerically observed with Eq. (40). Since Eq. (51) is the result of the approximation that the diagonal element $l=l^{\prime}=0$ is neglected, we should expect corrections depending on the chain length $N$. These finitesize corrections are responsible for the fine structures seen in Fig. 1. ${ }^{34}$ Moreover, the eigenvalues (51) have a nonzero imaginary part corresponding to damped oscillatory behavior in the dynamics. They therefore describe the short-time decay of the quantum coherences of the subsystem. These eigenvalues characterize the decoherence of the quantum dynamics on the chain and are denoted by the superscript $(C)$ in reference to their role in the decay of the quantum coherences.

We notice that the damping rates of the coherent eigenvalues (51) are larger than or equal to those of the diffusive eigenvalues (47), as observed in Fig. 1. According to Eq. (12), the damping rates of the coherent eigenvalues are, contrary to the diffusive rates, proportional to the temperature of the environment and to the square of the coupling parameter.

The theoretical analysis here above provides us with analytical expressions for the two families of eigenvalues seen in the previous numerical analysis. They also explain the crossover between the diffusive and the nondiffusive regimes seen in Fig. 1.

\section{E. Crossover between the diffusive and nondiffusive regimes}

We see in Fig. 1(a) that the diffusive eigenvalues (47) exist for all the values of the wave number $q$ in the case $A / Q \lambda^{2}<\frac{1}{2}$, which implies $\beta<\frac{1}{2}$. However, if $A / Q \lambda^{2}>\frac{1}{2}$, the diffusive eigenvalues disappear as expected for the values of the wave number corresponding to $\beta>\frac{1}{2}$. This situation is observed in Fig. 1(b). According to Eq. (47), the critical value of the wave number where the diffusive branch meets the other family of coherent eigenvalues is equal to

$$
q_{\mathrm{c}}=2 \arcsin \frac{Q \lambda^{2}}{2 A} .
$$

If this critical wave number is smaller than the smallest nonvanishing discrete value (30) allowed by the finiteness of the chain, the diffusive branch of the spectrum is reduced to the sole eigenvalue $q=0$, as seen in Fig. 1(c). Consequently, the crossover between the diffusive and nondiffusive regimes happens at

$$
q_{\mathrm{c}}=\frac{2 \pi}{N}
$$

Hence the diffusive branch disappears when the value of $\beta$ for the first nonzero eigenvalue corresponding to $q=2 \pi / N$ is larger than the critical value $\beta_{\mathrm{c}}=\frac{1}{2}$. This happens when the coupling parameter exceeds the critical value given by

$$
\lambda_{\mathrm{c}}=\sqrt{\frac{2 A}{Q} \sin \frac{\pi}{N}}^{N>5} \simeq \sqrt{\frac{2 A \pi}{Q N}} .
$$

This condition can be translated in terms of the critical size of the chain,

$$
N_{\mathrm{c}}=\frac{\pi}{\arcsin \frac{Q \lambda^{2}}{2 A}} \simeq \frac{2 \pi A}{Q \lambda^{2}}
$$

below which diffusive behavior no longer exists and the relaxation is only controled by simple decay processes with rates approximately given by $2 Q \lambda^{2}$. We point out that the diffusion always exists in the infinite-system limit $(N \rightarrow \infty)$ in which case $\lambda_{c}$ can be arbitrarily small. The critical size (55) is proportional to the width $4 A$ of the energy band and inversely proportional to the temperature according to Eq. (12). The higher the temperature, the smaller are the chains 
where the crossover from simple decay to diffusion happens.

We notice that a similar crossover would also exist in a finite open chain with reflecting boundary conditions at its two ends.

\section{DYNAMICAL ANALYSIS}

We have described in the last section the crossover between the nondiffusive and diffusive regimes in terms of the spectrum of the Redfield superoperator. We now need to discuss the dynamical implications of our spectral analysis.

\section{A. Theoretical predictions}

The spectrum of the Redfield superoperator determines the full dynamics of the subsystem interacting with its environment. In fact, projecting Eq. (16) in the site basis and using the Bloch property (22), we can write

$$
\rho_{l l^{\prime}}(t)=\sum_{q, \theta} \mathrm{e}^{s_{q \theta} t+i l q} c_{q \theta}(0) \rho_{0, l^{\prime}-l}^{q \theta}
$$

We see that the imaginary part of the eigenvalues $s_{q \theta}$ will generate oscillations in the dynamics and that the real part of these eigenvalues will cause a damping. The contribution to the long-time dynamics from the modes of the Redfield superoperator corresponding to the eigenvalues $s_{q \theta}$ which have a large real part is very small. Only the modes corresponding to eigenvalues with a small real part will significantly contribute to the long-time dynamics and therefore to the transport property of the system. Here, we mainly want to focus on the long-time dynamics.

As pointed out in Sec. III E, the subsystem evolves in a nondiffusive regime if the chain is small enough $\lambda<\lambda_{\mathrm{c}}$. In this regime, there is no diffusive eigenvalue so that the longtime dynamics is dominated by the eigenvalues (51) describing the decay of the quantum coherences. The relaxation rates associated with these eigenvalues are given by their real part, as seen in Sec. III C. Since these eigenvalues have a nonvanishing imaginary part, the slowest modes relax exponentially but with oscillations. These damped oscillations are reminiscent of a similar behavior in the spin-boson model. ${ }^{1}$ We can thus interpret these damped oscillations as due to the damping of the quantum coherences.

For a sufficiently large chain $\lambda>\lambda_{c}$, these damped oscillations disappear because the eigenvalues of the Redfield superoperator with the smallest real part are now the diffusive eigenvalues (47). In this case, the lowest relaxation rate is given by Eq. (39) with the diffusion coefficient (48),

$$
s^{(\mathrm{D})} \simeq-D q^{2}=-D\left(\frac{2 \pi}{N}\right)^{2}=-\frac{4 \pi^{2} A^{2}}{Q \lambda^{2} N^{2}},
$$

because the wave number of the slowest nontrivial mode takes the value $q=2 \pi / N$ and is inversely proportional to the length $N$ of the chain. The signature of the diffusive regime is therefore that the relaxation rate scales as $N^{-2}$.

This behavior is consistent with the famous Einstein relation for the definition of the diffusion coefficient. Indeed, we can define the position operator of the subsystem $\hat{x}$ by

$$
\left\langle l|\hat{x}| l^{\prime}\right\rangle=l \delta_{l l^{\prime}} .
$$

The variance of the position is therefore given by

$$
\left\langle\hat{x}^{2}\right\rangle(t)=\sum_{l=0}^{N-1} l^{2} \rho_{l l}(t),
$$

where, using Eq. (56),

$$
\rho_{l l}(t)=\sum_{q, \theta} a_{q \theta}(0) \mathrm{e}^{s_{q \theta} t+i l q},
$$

with the newly defined coefficients $a_{q \theta}(0)=c_{q \theta}(0) \rho_{00}^{q \theta}$. In the long-time limit and for a large enough chain, only the diffusive eigenvalues $s^{(\mathrm{D})}$ of spectrum will significantly contribute to the dynamics and one can write

$$
\rho_{l l}(t)=\sum_{q \theta} a_{q \theta}(0) \mathrm{e}^{s_{q \theta}}{ }^{t+i l q},
$$

where $s_{q \theta}=-D q^{2}$. Using $q_{j}=j 2 \pi / N, \Delta q=q_{j+1}-q_{j}=2 \pi / N$, and $f\left(q_{j}\right)=N a_{q_{j} \theta}(0)$, we get

$$
\rho_{l l}(t) \stackrel{t \rightarrow \infty}{=} \frac{1}{2 \pi} \sum_{j=0}^{N-1} \Delta q f\left(q_{j}\right) \mathrm{e}^{-D q_{j}^{2} t+i l q_{j}},
$$

which becomes in the limit of an arbitrarily large chain

$$
\rho_{l l}(t) \stackrel{N, t \rightarrow \infty}{=} \frac{1}{2 \pi} \int_{-\infty}^{+\infty} d q f(q) \mathrm{e}^{-D q^{2} t+i l q} .
$$

If we choose an initial condition of the density function centered on a given site, $f(q)=1$, and

$$
\rho_{l l}(t)=\frac{N, t \rightarrow \infty}{\sqrt{4 \pi D t}} .
$$

Finally, we recover the well-known Einstein relation for the diffusion coefficient:

$$
\left\langle\hat{x}^{2}\right\rangle(t) \stackrel{N, t \rightarrow \infty}{=} 2 D t .
$$

This demonstration is also a justification for calling diffusive the eigenvalues (47).

\section{B. Numerical results}

The previous theoretical predictions are confirmed by the numerical integration of the Redfield equation.

Let us define the quantity we compute. Equation (56) can be written as

$$
\rho_{l l}(t)=\sum_{q \theta} \mathrm{e}^{s_{q \theta} t+i l q} a_{q \theta}(0),
$$

where $a_{q \theta}(0)=c_{q \theta}(0) \rho_{00}^{q \theta}$. If we introduce

$$
\widetilde{\rho}\left(q^{\prime}, t\right)=\sum_{l=0}^{N-1} \rho_{l l}(t) \mathrm{e}^{-i l q^{\prime}},
$$

we find using Eq. (5) that 

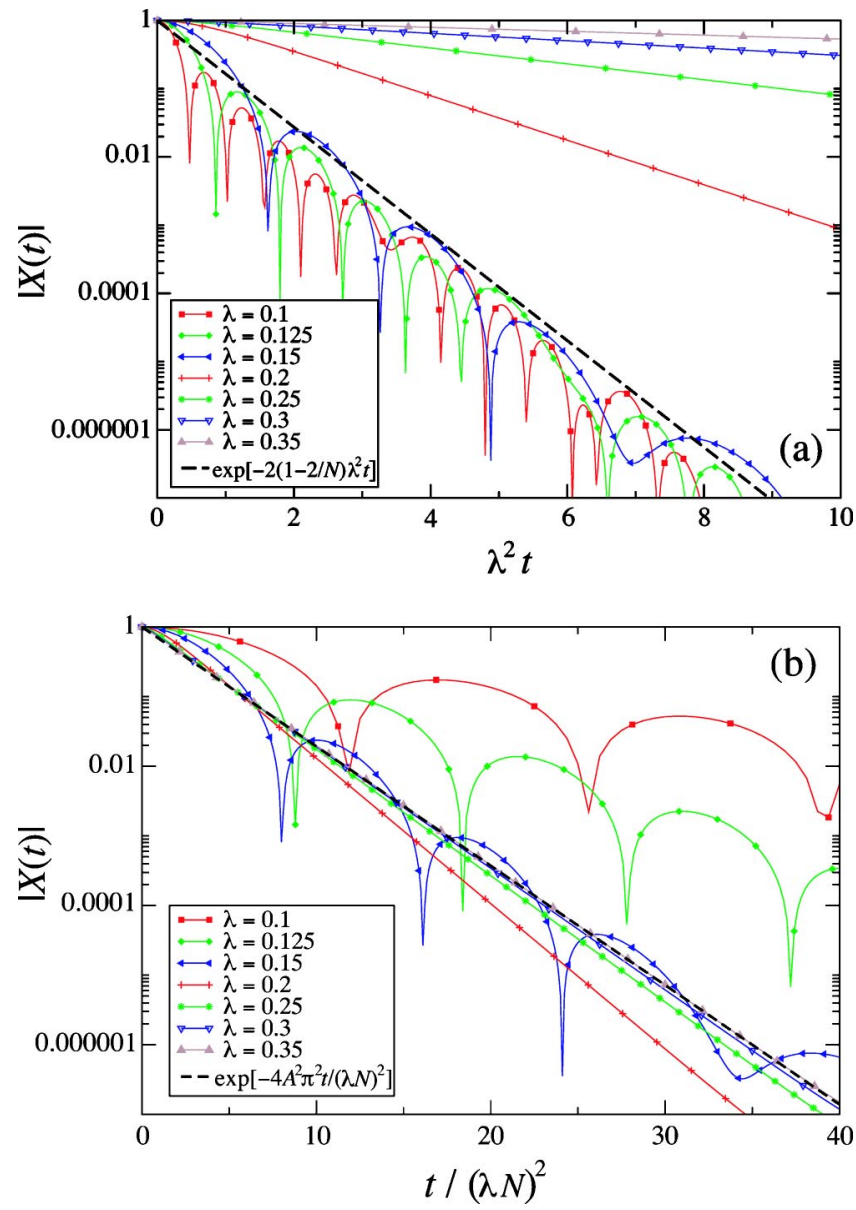

FIG. 2. (Color online) Crossover between the nondiffusive and diffusive regimes for a chain of size $N=20$ and parameters $E_{0}=0$, $A=0.1$, and $Q=1$. The crossover occurs around the critical value $\lambda_{c}=0.177$ given by Eq. (54). (a) The quantity $|X(t)|$ versus the rescaled time $\lambda^{2} t$ in order to identify the nondiffusive regime. (b) The quantity $|X(t)|$ versus the rescaled time $t /(\lambda N)^{2}$ in order to identify the diffusive regime. As predicted, the nondiffusive regime manifests itself if $\lambda<\lambda_{c}=0.177$ and the diffusive regime if $\lambda>\lambda_{c}$ $=0.177$. The quantity $|X(t)|$ is defined in Eq. (69).

$$
\widetilde{\rho}\left(q^{\prime}, t\right)=N \sum_{\theta} a_{q^{\prime} \theta}(0) \mathrm{e}^{s q^{\prime} \theta}
$$

The evolving quantity we have computed is

$$
|X(t)|=\left|\tilde{\rho}\left(q_{1}, t\right)\right|,
$$

where we recall that $q_{1}=2 \pi / N$. We consider that the subsystem has initial conditions given by

$$
\rho_{l l^{\prime}}(0)=\delta_{l l^{\prime}} \delta_{l 0}
$$

This means that $\Sigma_{\theta} a_{q \theta}(0)=1 / N$. The quantity $X(t)$ is initially equal to unity $[X(0)=1]$ and tends to zero after long times $[X(\infty)=0]$ in an exponential way determined by the smallest nonvanishing real part of the eigenvalues $s_{q_{1}}$.

Figures 2(a) and 2(b) depict the relaxation curves of $|X(t)|$ for different values of the coupling parameter $\lambda$ but to the same chain size $N$. In order to determine the regime, we use two different scalings with respect to the time. Figure 2(a)

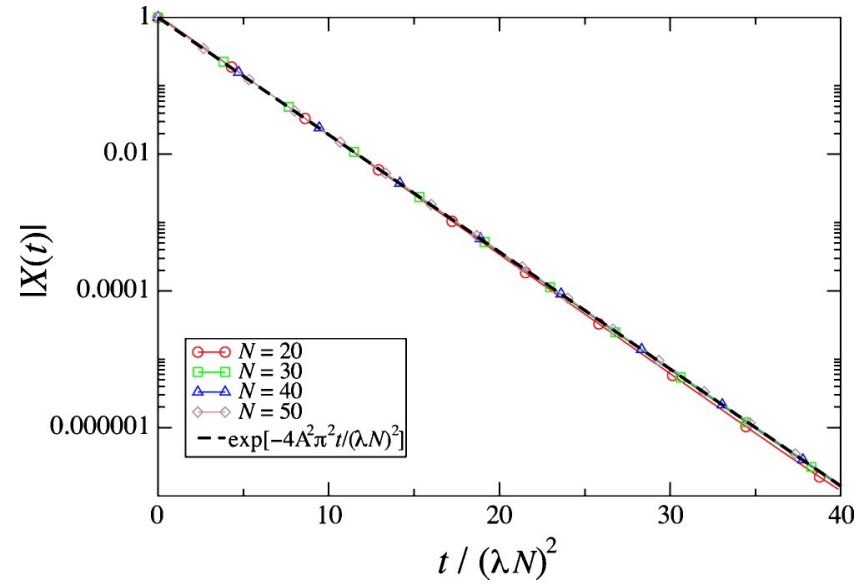

FIG. 3. (Color online) The quantity $|X(t)|$ defined by Eq. (69) versus the rescaled time $t /(\lambda N)^{2}$ for various sizes $N=20-50$ of the chain. The parameters are $E_{0}=0, A=0.1, Q=1$, and $\lambda=0.3$. Here, the system is always in the diffusive regime because $N>N_{c}=6.73$.

has the scaling $\lambda^{2} t$, which is characteristic of the nondiffusive regime. Instead, Fig. 2(b) has the scaling $t /(\lambda N)^{2}$, which is characteristic of the diffusive regime. Therefore, the curves clustering in Fig. 2(a) correspond to values of the coupling parameter such that $\lambda<\lambda_{\mathrm{c}}$ (in the nondiffusive regime) and they relax with damped oscillations at rates given by Eq. (40). On the other hand, the curves clustering in Fig. 2(a) correspond to values of the coupling parameter such that $\lambda>\lambda_{\mathrm{c}}$ (in the diffusive regime) and they relax exponentially without oscillations at a rate given by Eq. (57).

Figure 3 depicts the relaxation curves for chains of different sizes $N$ but the same value of the coupling parameter. Here, the chain is in the diffusive regime as evidenced by the clustering of the different curves with the time scaling $t /(\lambda N)^{2}$. This figure numerically demonstrates the property of diffusion for $\lambda>\lambda_{\mathrm{c}}$ or $N>N_{\mathrm{c}}$.

An important remark is that the time scaling of the nondiffusive regime is naturally expected from perturbation theory. Indeed, according to perturbation theory, the relaxation rates are proportional to the square of the coupling parameter $\lambda$. This is consistent with the fact that the Redfield quantum master equation is obtained by second-order perturbation theory in the coupling parameter from the complete von Neumann equation for the subsystem interacting with its environment. It is quite remarkable that the time scaling of the relaxation is completely different in the diffusive regime where the relaxation rate depends on the inverse of the square of the coupling parameter.

In Figs. 4-6 we have represented the time evolution of the probability $P=\rho_{l l}(t)$ to occupy a given site of the subsystem for three different values of the coupling parameter. In Fig. 4, the coupling parameter is zero and the time evolution of the subsystem is purely Hamiltonian. The wave packet follows a ballistic motion along the chain which forms a closed loop due to the periodic boundary conditions. At long times, the evolution presents wavy interferences showing the coherent character of the dynamics in this uncoupled case. In Fig. 5, the coupling parameter is such that the subsystem relaxes in the nondiffusive regime. The dy- 
(a)

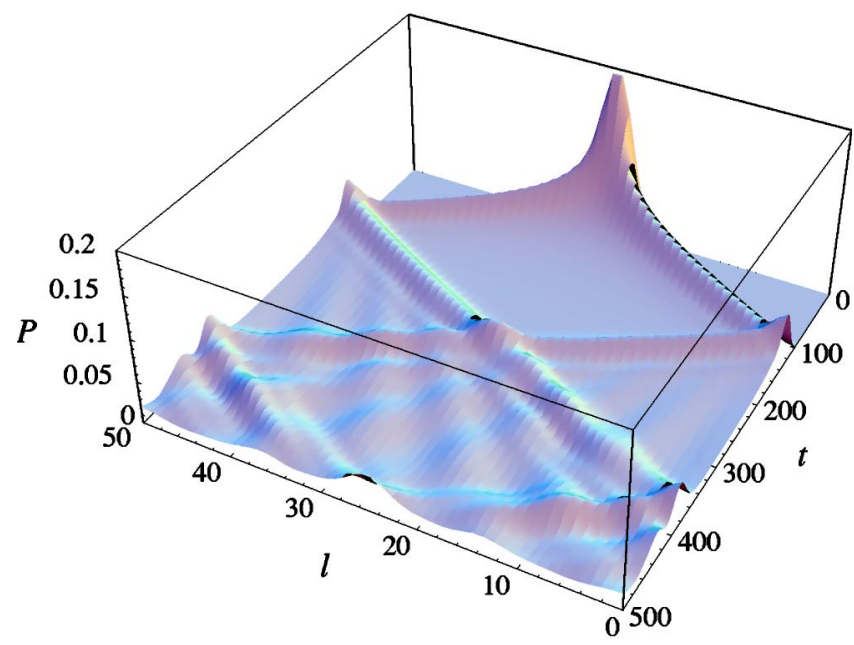

(b)

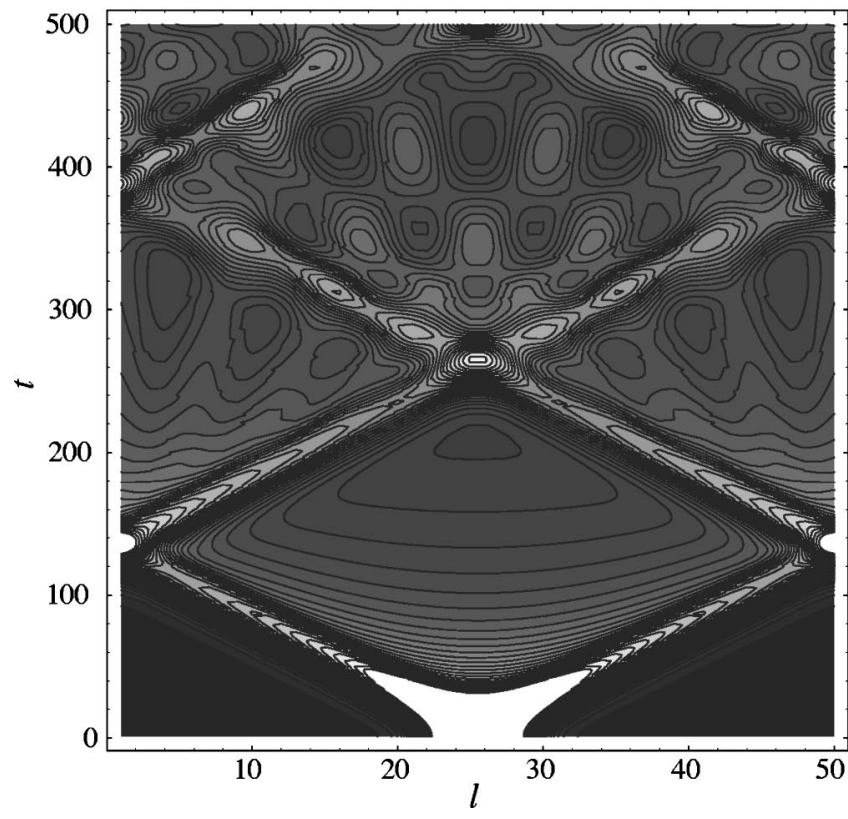

FIG. 4. (Color online) Representation of the time evolution of the probability $P=\rho_{l l}(t)$ to occupy a given site of the subsystem. The initial conditions correspond to a Gaussian wave packet of standard deviation 2 centered on the site $l=25$. The parameter values are: $N=50, E_{0}=0, A=0.1, Q=1$, and therefore $\lambda_{c}=0.1121$. The system is isolated $(\lambda=0)$ and the time evolution is thus a pure Hamiltonian multiperiodic dynamics. The center of the wave packet travels ballistically around the chain which forms a closed loop.

namics still presents wavy interferences, but they are damped by the dissipation to the environment. We notice that the center of the wave packet still travels along the chain but it is damped. Finally, in Fig. 6, the coupling parameter is now such that the subsystem relaxes in the diffusive regime. The dynamics of the subsystem is no longer coherent and the wavy interferences have disappeared. In this case, the center of the wave packet no longer moves along the chain but that it spreads diffusively. (a)

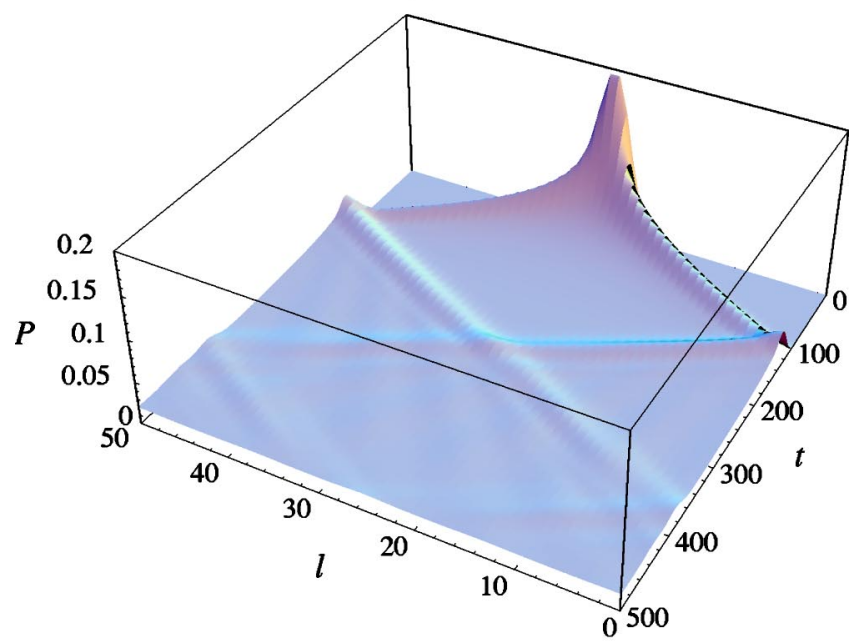

(b)

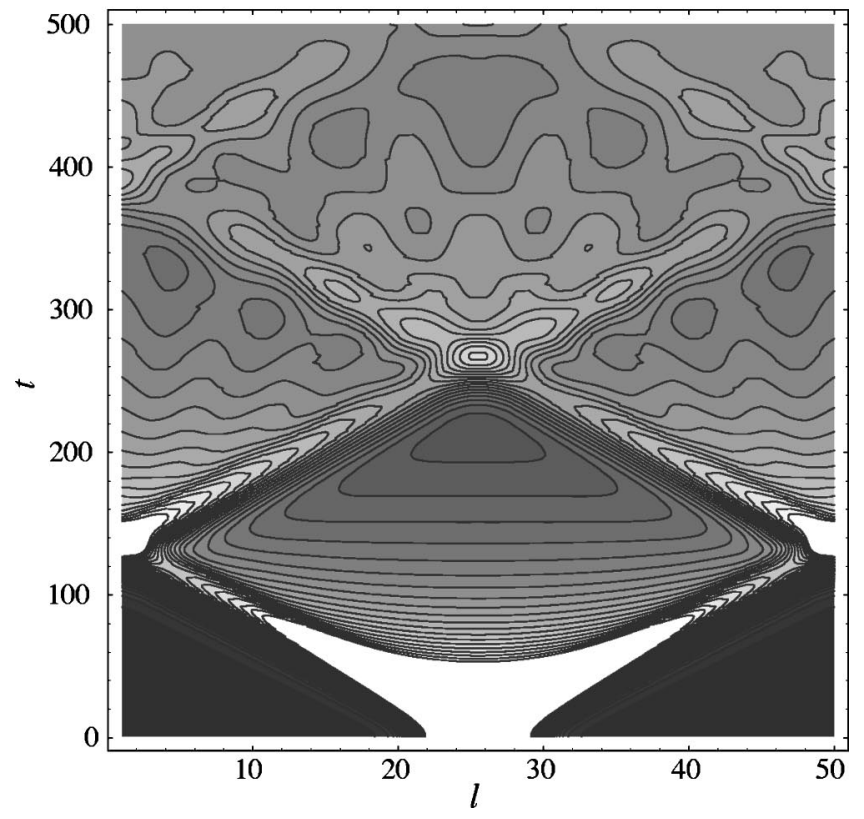

FIG. 5. (Color online) Representation of the time evolution of the probability $P=\rho_{l l}(t)$ to occupy a given site of the subsystem. The situation is the same as in Fig. 4, except that the system is now interacting with its environment with the coupling parameter $\lambda$ $=0.05$. The system therefore relaxes in the nondiffusive regime $(\lambda$ $<\lambda_{c}=0.1121$ ) and the time evolution is a damped multiperiodic dynamics. However, the center of the wave packet keeps traveling ballistically around the chain.

\section{CONCLUSIONS}

In the present paper, we have shown how the transport property of diffusion can emerge in a quantum chain interacting with a thermal environment. For this purpose, we have considered a simple model in which a tight-binding Hamiltonian with a single energy band is coupled to a thermal environment. The coupling is characterized by correlation functions which decay on time scales longer than the thermal time scale, but shorter than the subsystem time scale. The reduced dynamics of the subsystem is described by a Red- 
(a)

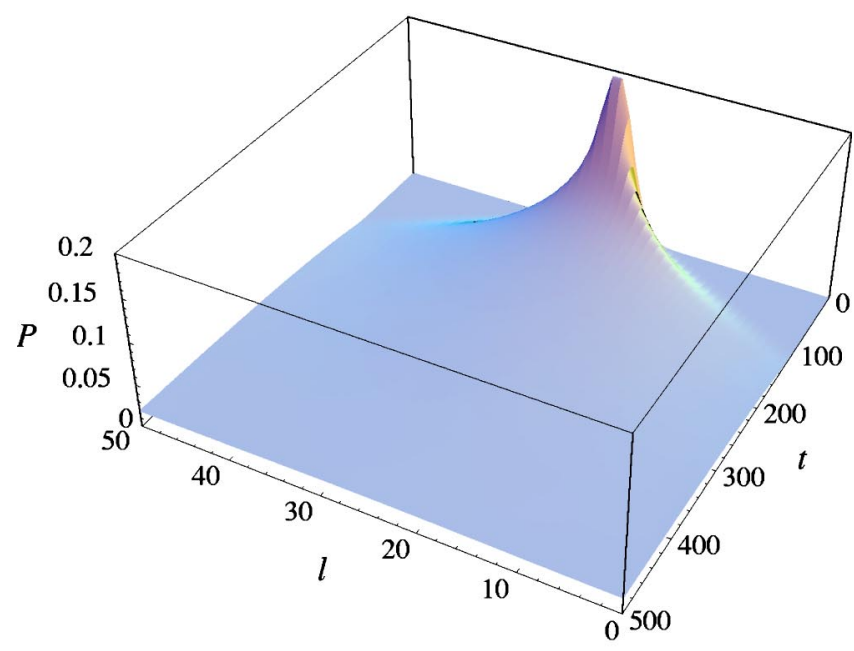

(b)

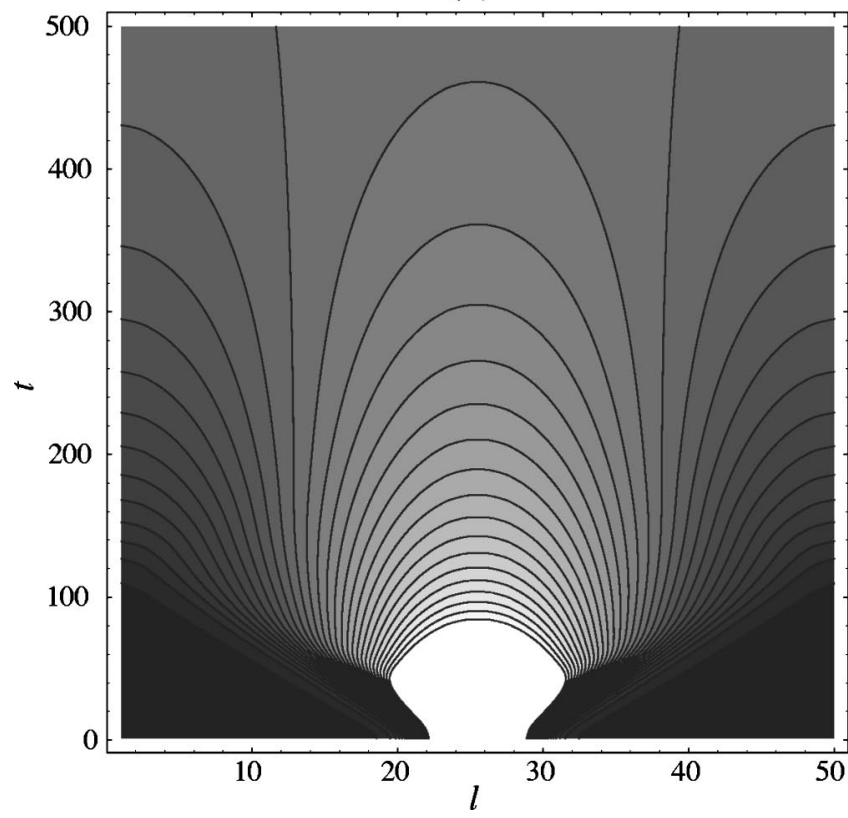

FIG. 6. Representation of the time evolution of the probability $P=\rho_{l l}(t)$ to occupy a given site of the subsystem. The situation is the same as in Fig. 5, but the coupling between the system and its environment is stronger $(\lambda=0.15)$. The system here relaxes in the diffusive regime $\left(\lambda>\lambda_{c}=0.1121\right)$. The multiperiodicity has disappeared, the center of the wave packet does not move, and a diffusive spreading of the wave packet can be observed.

field quantum master equation which takes, for such environments, a Lindblad form. Thanks to the invariance under spatial translations, we can apply the Bloch theorem to the density matrix of the subsystem. In this way, we succeeded in diagonalizing the Redfield superoperator. The eigenvalues control the time evolution of the subsystem and its relaxation to the thermodynamic equilibrium. Two kinds of eigenvalues were obtained: the eigenvalues giving the dispersion relation of diffusion along the one-dimensional subsystem and other eigenvalues which describe the decay of the quantum coherences-i.e., the process of decoherence in the subsystem.
The properties of the system depend on the length $N$ of the one-dimensional chain, on the width $4 A$ of the energy band of the unperturbed tight-binding Hamiltonian, and on the intensity $Q$ of the environmental noise multiplied in the combination $Q \lambda^{2}$ with the square of the coupling parameter $\lambda$ of perturbation theory.

We discovered that, for a finite chain, there are two regimes depending on the chain length $N$ and the physical parameters $A$ and $Q \lambda^{2}$.

For finite chains smaller than a critical size $N_{\mathrm{c}}$ given by Eq. (55), there is a nondiffusive regime characterized by a time evolution with oscillations damped by decay rates proportional to $Q \lambda^{2}$. The oscillations come from the time evolution of the quantum coherences at the Bohr frequencies. They are damped due to the coupling with the environment. This nondiffusive regime exists if the coupling parameter is smaller than a critical value which is inversely proportional to the square root of the chain size $N, \lambda<\lambda_{\mathrm{c}}=O\left(N^{-1 / 2}\right)$.

Longer chains evolve in the diffusive regime with a monotonic decay on long times at a rate controled by a diffusion coefficient. In this regime, the slower relaxation mode relaxes exponentially in time with the scaling $t /(\lambda N)^{2}$.

In the limit of an infinite chain $N \rightarrow \infty$ and for a nonvanishing coupling parameter $Q \lambda^{2}$, the nondiffusive regime disappears and the system always diffuses.

According to Eq. (48), the diffusion coefficient is proportional to the square of the width $4 A$ of the energy band and inversely proportional to the intensity $Q \lambda^{2}$ of the environmental noise. We are thus in the presence of a mechanism of diffusion in which the quantum tunneling of the particle from site to site is perturbed by the environmental fluctuations. For an Ohmic coupling to the environment, the constant $Q$ is proportional to the temperature and therefore the diffusion coefficient is inversely proportional to the temperature. By using Einstein's relation between the diffusion coefficient and the conductivity, this latter is inversely proportional to the square of the temperature as it is the case in many circumstances. ${ }^{28,35,36}$ In this regard, the model used as a vehicle of the present study present many typical features of quantum diffusion and conductivity.

In conclusion, we believe that the crossover between the nondiffusive and diffusive regimes we have obtained in the present paper is a general phenomenon of a whole class of quantum systems sustaining transport of quantum particles or charges such as molecular wires or carbon nanotubes in the form of closed loops. The present results provide an understanding of the emergence of diffusion out of quantum coherent behavior as the size of the loop increases.

\section{APPENDIX A: TEMPERATURE DEPENDENCE OF THE ENVIRONMENT CORRELATION FUNCTION}

As a consequence of the quantum fluctuation-dissipation theorem, the environment correlation function can be expressed in terms of the spectral strength $J(\omega)$ according to

$$
\alpha(t)=\int_{0}^{\infty} d \omega J(\omega)\left(\operatorname{coth} \frac{\omega}{2 k_{\mathrm{B}} T} \cos \omega t-i \sin \omega t\right) .
$$

The Fourier transform of the environment correlation function, which is given by 


$$
\widetilde{\alpha}(\omega)=\int_{-\infty}^{+\infty} \frac{d t}{2 \pi} \mathrm{e}^{i \omega t} \alpha(t),
$$

is therefore related to the spectral strength by

$$
\widetilde{\alpha}(\omega)=\frac{1}{2} \operatorname{sgn}(\omega) J(|\omega|)\left(\operatorname{coth} \frac{\omega}{2 k_{\mathrm{B}} T}+1\right),
$$

where $\operatorname{sgn}(\omega)$ denotes the sign of $\omega$. Empirical forms for the spectral strength are often used in the literature ${ }^{1,17}$ such as

$$
J(|\omega|)=K|\omega|^{\gamma} \mathrm{e}^{-|\omega| / \omega_{\mathrm{b}}},
$$

where $\omega_{\mathrm{b}}$ is a cutoff frequency and $K$ a constant. The environment is called Ohmic if $\gamma=1$, sub-Ohmic if $\gamma<1$, and super-Ohmic if $\gamma>1$. The correlation time $\tau_{\mathrm{b}}$, defined as the time over which the environment correlation function decays to zero, is given by the inverse of the cutoff frequency by $\tau_{\mathrm{b}}=1 / \omega_{\mathrm{b}}$.

If one assumes that the thermal time of the environment $t_{\mathrm{th}}=1 / k_{\mathrm{B}} T$ is shorter than the correlation time $\tau_{\mathrm{b}}$, simplifications occur. The imaginary part of the environment correlation function becomes negligible compared to its real part and Eq. (A1) becomes

$$
\alpha(t) \simeq 2 k_{\mathrm{B}} T \int_{0}^{\infty} d \omega \frac{J(\omega)}{\omega} \cos \omega t .
$$

Notice that the environment correlation function is now symmetric in time $\alpha(t)=\alpha(-t)$. The Fourier transform of the correlation function $(\mathrm{A} 3)$ reduces to

$$
\widetilde{\alpha}(\omega) \simeq k_{\mathrm{B}} T \frac{J(|\omega|)}{|\omega|} .
$$

Notice that the zero-frequency limit of the Fourier transform of the correlation function,

$$
\lim _{\omega \rightarrow 0} \widetilde{\alpha}(\omega)=\lim _{\omega \rightarrow 0} K k_{\mathrm{B}} T|\omega|^{\gamma-1}
$$

is well defined and nonvanishing only in the case of an Ohmic spectral strength $\gamma=1$.

As a consequence, the time integral of the correlation function defined by Eq. (11) is equal to

$$
Q \equiv \frac{1}{2} \int_{-\infty}^{+\infty} d \tau \alpha(\tau)=\pi \widetilde{\alpha}(0)=\pi K k_{\mathrm{B}} T
$$

and is therefore proportional to the temperature.

\section{APPENDIX B: TEMPERATURE DEPENDENCE OF THE DIFFUSION COEFFICIENT AND THE CONDUCTIVITY}

Using Eqs. (12) and (48), the diffusion coefficient of the present model can be written with its explicit temperature dependence:

$$
D=\frac{A^{2}}{\pi K \lambda^{2} k_{\mathrm{B}} T} .
$$

The conductivity $\sigma$ can also be obtained using Einstein's relation

$$
D=\frac{\sigma}{e^{2}}\left(\frac{\partial \mu}{\partial n}\right)_{T},
$$

where $e$ is the electric charge of the carriers, $n$ their density, and $\mu$ their chemical potential. Assuming a low density of carriers, the chemical potential depends on the density according to

$$
\mu=\mu^{0}(T)+k_{\mathrm{B}} T \ln \frac{n}{n^{0}},
$$

so that the conductivity is given by

$$
\sigma=\frac{e^{2} n}{k_{\mathrm{B}} T} D=\frac{e^{2} A^{2} n}{\pi K \lambda^{2}\left(k_{\mathrm{B}} T\right)^{2}} .
$$

Therefore, the conductivity decreases as $T^{-2}$ with the temperature. We notice that similar dependences are also obtained for the electric conductivity in conducting polymers ${ }^{28}$ and in Fermi liquids. ${ }^{35,36}$ This inverse power law is due to the existence of a single conduction band in the model. The transport is therefore confined in this band and no thermally activated transport process can occur. The diffusive transport phenomenon can be viewed as the result of the tunneling of the particle through the potential barriers of the system. This tunneling is more and more affected as the temperature increases.

We expect that a crossover would occur to a regime where the diffusion coefficient has a temperature dependence of Arrhenius type if one considered a system with at least two conduction bands. In such systems, we should find that a crossover between the quantum tunneling regime and a thermally activated transport regime when $k_{\mathrm{B}} T$ becomes comparable to the energy spacing between the two bands. ${ }^{12,17,23,24,26,27}$

\section{ACKNOWLEDGMENTS}

The authors thank Professor G. Nicolis for support and encouragement in this research. M.E. is supported by the Fond pour la formation à la Recherche dans 1'Industrie et dans l'Agriculture and P.G. by the National Fund for Scientific Research (F.N.R.S. Belgium). This research is financially supported by the "Communauté française de Belgique" ("Actions de Recherche Concertées," Contract No. 04/09312), the National Fund for Scientific Research (F.N.R.S. Belgium, F.R.F.C., Contract Nos. 2.4542.02 and 2.4577.04), and the U.L.B. 
${ }^{1}$ A. J. Leggett, S. Chakravarty, A. T. Dorsey, M. P. A. Fisher, A. Garg, and W. Zwerger, Rev. Mod. Phys. 59, 1 (1987); 67, 725(E) (1995).

${ }^{2}$ A. G. Redfield, IBM J. Res. Dev. 1, 19 (1957).

${ }^{3}$ N. G. van Kampen, Stochastic Processes in Physics and Chemistry, 2nd ed. (North-Holland, Amsterdam, 1997).

${ }^{4}$ C. W. Gardiner and P. Zoller, Quantum Noise, 2nd ed. (Springer, Berlin, 2000).

${ }^{5}$ C. Cohen-Tannoudji, J. Dupont-Roc, and G. Grynberg, Processus d'Interaction entre Photons et Atomes (CNRS Editions, Paris, 1996).

${ }^{6}$ P. Gaspard and M. Nagaoka, J. Chem. Phys. 111, 5668 (1999).

${ }^{7}$ N. W. Ashcroft and N. D. Mermin, Solid State Physics (Saunders College, Fort Worth, 1976).

${ }^{8}$ S. Chandrasekhar, Rev. Mod. Phys. 15, 1 (1943).

${ }^{9}$ P. Résibois and M. De Leener, Classical Kinetic Theory of Fluids (Wiley, New York, 1977).

${ }^{10}$ H. van Beijeren, Rev. Mod. Phys. 54, 195 (1982).

${ }^{11} \mathrm{R}$. Haase, Thermodynamic of Irreversible Processes (Dover, New York, 1990).

${ }^{12}$ T. Holstein, Ann. Phys. (N.Y.) 8, 325 (1959); 8, 343 (1959).

${ }^{13}$ A. O. Caldeira and A. J. Leggett, Physica A 121, 587 (1983).

${ }^{14}$ M. P. A. Fisher and W. Zwerger, Phys. Rev. B 32, 6190 (1985).

${ }^{15}$ M. Sassetti, M. Milch, and U. Weiss, Phys. Rev. A 46, 4615 (1992).

${ }^{16}$ M. Sassetti, H. Schomerus, and U. Weiss, Phys. Rev. B 53, R2914 (1996).

${ }^{17}$ U. Weiss, Quantum Dissipative Systems, 2nd ed. (World Scientific, Singapore, 2000).
${ }^{18}$ C. H. Mak and R. Egger, Phys. Rev. E 49, 1997 (1994).

${ }^{19}$ Y.-C. Chen, J. L. Lebowitz, and C. Liverani, Phys. Rev. B 40, 4664 (1989).

${ }^{20}$ Y.-C. Chen and J. L. Lebowitz, Phys. Rev. B 46, 10743 (1992).

${ }^{21}$ Y.-C. Chen and J. L. Lebowitz, Phys. Rev. B 46, 10751 (1992).

${ }^{22}$ D. Cohen, Phys. Rev. E 55, 1422 (1997).

${ }^{23}$ V. G. Storchak and N. V. Prokof'ev, Rev. Mod. Phys. 70, 929 (1998).

${ }^{24}$ V. Pouthier and J. C. Light, J. Chem. Phys. 113, 1204 (2000).

${ }^{25}$ V. Pouthier, J. C. Light, and C. Girardet, J. Chem. Phys. 114, 4955 (2001).

${ }^{26}$ R. DiFoggio and R. Gomer, Phys. Rev. B 25, 3490 (1982).

${ }^{27}$ L. J. Lauhon and W. Ho, Phys. Rev. Lett. 85, 4566 (2000).

${ }^{28}$ A. J. Heeger, S. Kivelson, J. R. Schrieffer, and W. P. Su, Rev. Mod. Phys. 60, 781 (1988).

${ }^{29}$ R. Saito, G. Dresselhaus, and M. S. Dresselhaus, Physical Properties of Carbon Nanotubes (Imperial College Press, London, 1998).

${ }^{30}$ J. M. Jean, J. Chem. Phys. 101, 10464 (1994).

${ }^{31}$ M. Mehring, Principles of High-Resolution NMR in Solids (Springer, Berlin, 1983).

${ }^{32}$ G. C. Schatz and M. A. Ratner, Quantum Mechanics in Chemistry (Prentice-Hall, Englewood Ciffs, NJ, 1993).

${ }^{33}$ G. Lindblad, Commun. Math. Phys. 48, 119 (1976).

${ }^{34} \mathrm{M}$. Esposito and P. Gaspard, cond-mat/0505217.

${ }^{35}$ D. Pines and P. Nozières, The Theory of Quantum Liquids (Addison-Wesley, Redwood City, CA, 1989).

${ }^{36}$ G. Mahan, Many Particles Physics (Plenum, New York, 1990). 